\title{
Delivery of HIV-1 Nef linked to heat shock protein 27 using a cationic polymer is more effective than cationic lipid in mammalian cells
}

\author{
Milani $\mathrm{A}^{1}$, Bolhassani $\mathrm{A}^{2}$, Heshmati $\mathrm{M}^{1}$ \\ Department of Hepatitis and AIDS, Pasteur Institute of Iran, Tehran, Iran. azam.bolhassani@yahoo.com
}

\begin{abstract}
BACKGROUND: Different adjuvants and delivery systems have been used to enhance the potency of DNA vaccines against viral diseases. Among them, heat shock proteins (HSPs) are stress proteins that have multiple roles such as chaperon activity and anti-apoptotic and adjuvant properties. The goal of this study was to compare the expression of HIV-1 Nef, Hsp27 and Hsp27-Nef genes transfected in HEK-293T mammalian cells by TurboFect and Lipofectamine as a cationic polymer and lipid, respectively.

METHODS: At first, the pEGFP eukaryotic vectors encoding HIV-1 Nef, Hsp27 and Hsp27-Nef genes were generated and transfected in HEK-293T using TurboFect and Lipofectamine delivery systems. Then, the expression of proteins was evaluated and compared using fluorescent microscopy, flow cytometry and western blotting $48 \mathrm{hr}$ after transfection.

RESULTS: The accuracy of the DNA constructs was confirmed on agarose gel electrophoresis to be $\sim 720$ bp, $\sim 648 \mathrm{bp}$, and $\sim 1368$ bp bands for Hsp27, Nef, and Hsp-Nef, respectively. The expression analysis in the transfected cells showed that the delivery of genes using TurboFect was significantly higher than that using Lipofectamine. Furthermore, transfection of Hsp27 gene was more effective than that of Nef gene using both delivery systems. Hsp27 linked to Nef could also increase its delivery and expression in HEK-293T cells. CONCLUSION: Generally, Hsp27 can be used as a suitable carrier in DNA vaccine design against HIV-1 infections (Fig. 5, Ref. 28). Text in PDF www.elis.sk.

KEY WORDS: small heat shock protein, HIV-1 Nef, Hsp27, gene delivery.
\end{abstract}

\section{Introduction}

Human immunodeficiency virus (HIV) weakens the immune system causing acquired immune deficiency syndrome (AIDS). The major cells targeted by HIV include macrophages, T-cells, and dendritic cells (DCs). Different receptors are required in this way, such as CD4 and CCR5 or CXCR4 on T-cells, galactocerebroside (Gal-C) on macrophage, and DC-SIG on DCs for viral spreading $(1,2)$. The HIV genome contains three major genes encoding fifteen viral proteins essential for the viral life cycle: a) gag, pol, env genes expressing enzymes, structural and envelope proteins, respectively; b) tat, rev genes encoding regulatory proteins, and c) $v p r, v i p, v p u$, and nef encoding accessory proteins (3). Therefore, researchers designed a way of stimulating the immune system by using viral genes as antigens. Nef protein (MW: $27 \mathrm{kDa}$ ) is expressed early in viral life cycle and has important roles includ-

${ }^{1}$ Department of Molecular and Cellular Sciences, Faculty of Advanced Sciences \& Technology, Pharmaceutical Sciences Branch, Islamic Azad University, Tehran, Iran, and ${ }^{2}$ Department of Hepatitis and AIDS, Pasteur Institute of Iran, Tehran, Iran

Address for correspondence: A. Bolhassani, PhD, Department of Hepatitis and AIDs, Pasteur Institute of Iran Tehran, Iran.

Phone: +98 2166465132 ing inhibition of CD4, MHC I, MHC II activity, stimulation of replication and viral infection, and induction of apoptosis in both uninfected and infected cells. Generally, the studies showed that the lack of Nef reduces the infectivity of the virus (4-9). Indeed, expression of Nef protein in the first phase of the infection, its existence during the period of infection, and having epitopes for T-cells are the advantages of Nef as a good antigen candidate for HIV vaccine development. However, recent findings indicated low permeability of the DNA constructs and their poor immunogenicity. Thus, such vaccines should be improved by delivery systems and adjuvants (10). There are three major tools for gene delivery including viral, physical and chemical methods. Due to some drawbacks of both viral and physical methods, a variety of chemical transfection systems were developed, such as lipids, cationic polymers and calcium phosphates. The cationic polymers (e.g., TurboFect) and lipids (e.g., lipofectamine) could generate compact and stable complexes with negatively charged nucleic acid molecules, protect DNA from degradation, and facilitate efficient DNA delivery into the cells $(11,12)$. On the other hand, an adjuvant enhances the immunity to an antigen in conjugate and complex forms. Heat shock proteins (HSPs) are highly conserved proteins that play main roles in maintaining protein homeostasis during cellular stress. They were classified based on their molecular weights including HSP90, HSP70, HSP60 and small HSPs. Many 
HSPs exhibited the regulatory and stimulatory roles on immune responses (13-15). Small Hsps are the members of HSP family with molecular weights from 12 to $43 \mathrm{kDa}$. These proteins have a similar structure such as $\mathrm{N}$-terminal domain, $\alpha$-crystalline, and C-terminal domain (16). Treatment of macrophage with the recombinant Hsp27 (rHsp27) led to an increase in the expression of both pro-inflammatory cytokines (e.g., IL-1 $\beta, \mathrm{TNF}-\alpha$ ), and anti-inflammatory cytokines (e.g., IL-10, GM-CSF) (17-19). Herein, we prepared DNA constructs of Hsp27, Nef, and Hsp27-Nef for evaluation of their expression in mammalian cell line. Then, these constructs were transfected to the HEK-293T cell line using TurboFect and Lipofectamine transfection regents and their expression were assessed by fluorescent microscopy, flow cytometry and western blotting. The data showed that both systems were suitable for delivery of DNA constructs in a certain dose, but the transfection efficiency of TurboFect system was higher than that of Lipofectamine.

\section{Materials and methods}

\section{Construction of the recombinant plasmids}

The full length of Hsp27 gene was prepared in a prokaryotic expression vector (pQE30, Biomatik Co., Canada). To generate pEGFP-Hsp27, Hsp27 fragment was subcloned into the pEGFPN3 eukaryotic expression vector in NheI/ SalI sites (Fermentas). HIV-1 Nef sequence was subcloned from $p U C-19$ into the pEGFPN1 in NheI/PstI sites. To make pEGFP-Hsp27-Nef, at first, pUCNef and pQE30-Hsp27 were digested by BamHI/PstI and BglII/ PstI, respectively. Then, Nef fragment was ligated to Hsp27 in pQE30-Hsp27 (BamHI and BglII are compatible enzymes) using T4 DNA Ligase (Fermentas). Finally, Hsp27-Nef fusion was cut from pQE-Hsp27-Nef, and subcloned into pEGFP-N1 using NheI/ PstI restriction endonucleases. The $E$. coli DH5 $\alpha$ competent cells were transformed by all plasmids by heat shock for 90 second at $42{ }^{\circ} \mathrm{C}$. The single clones were cultured overnight in Luria-Bertani (LB) broth at $37^{\circ} \mathrm{C}$. Finally, the recombinant plasmids were purified with plasmid DNA extraction mini kit (Yekta Tajhiz Azma, Iran) and confirmed by digestion and sequencing.

\section{Cell Culture}

Human Embryonic Kidney 293T cells (HEK-293T, Pasteur Institute of Iran) were grown in Dulbecco's Modified Eagle's Medium (DMEM) (Sigma), supplemented with 10\% fetal bovine serum (FBS, Gibco), pen/strep (100U/ml penicillin and $0.1 \mathrm{mg} / \mathrm{ml}$ Streptomycin, Gibco). The cell cultures were incubated at $37^{\circ} \mathrm{C}$ and $5 \% \mathrm{CO}_{2}$ conditions. One day before transfection, $5 \times 10^{5}$ cells were seeded into the 6 -well plates to achieve exponential growth phase.

\section{Transfection}

HEK-293T cells at 70-80\% confluency were transfected with pEGFP-Hsp27, pEGFP-Nef, pEGFP-Hsp27-Nef, and pEGFP-N1 as a positive control using TurboFect (Thermo scientific) and Lipofectamine ${ }^{\mathrm{TM}} 2000$ (Invitrogen) reagents. To generate TurboFectplasmid DNA complex, $7 \mu \mathrm{l}$ of TurboFect and $4 \mu \mathrm{g}$ of plasmid were mixed and incubated for 15 minutes at room temperature. Finally, the complexes were added dropwise to each well in serum-free
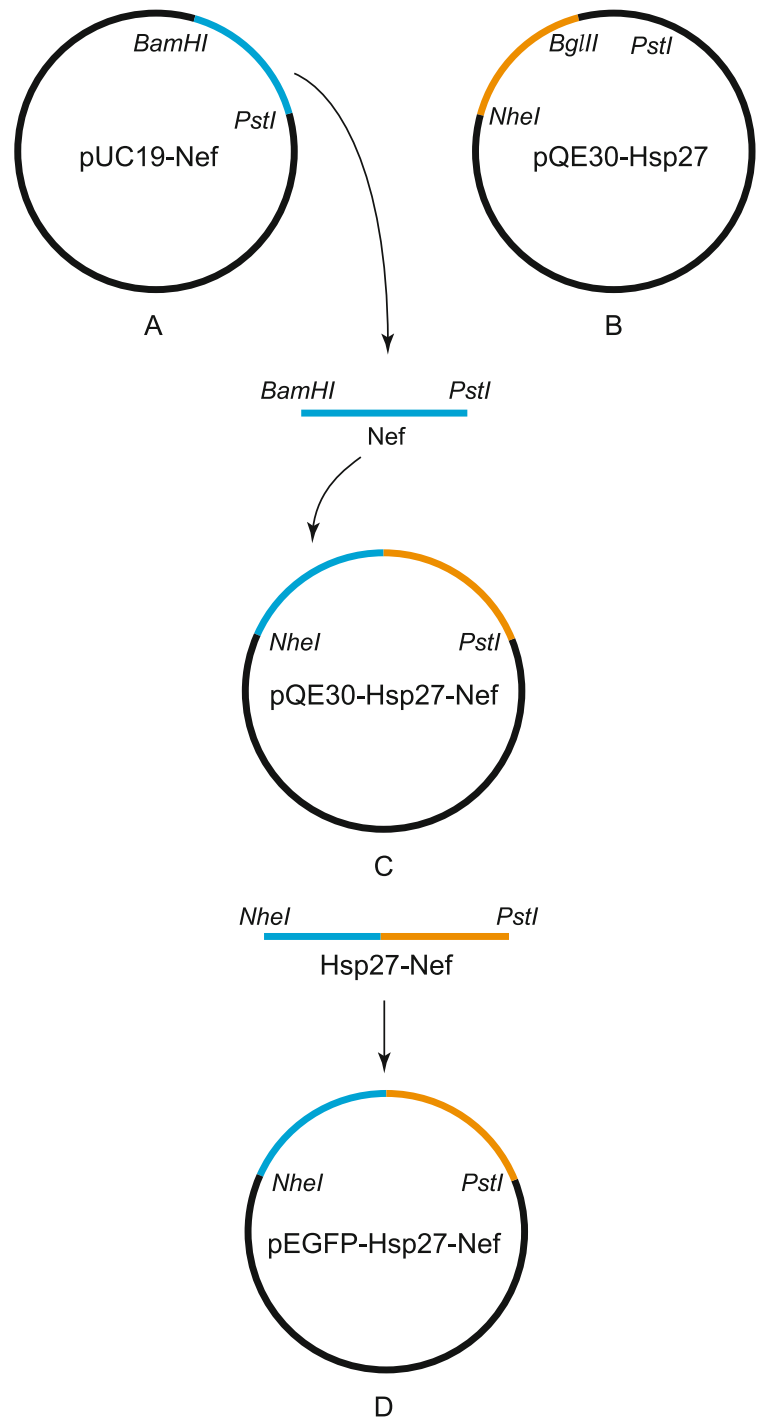

Fig. 1. The detailed process of pEGFP-Hsp27-Nef construct: A) Nef fragment was digested from pUC-19 with BamHI/PstI, B) pQE30Hsp27 was digested with BglII/PstI, C) Nef fragment was ligated into the linearized pQE-Hsp27 with T4 DNA ligase, D) Hsp27-Nef fusion was cut from $\mathrm{pQE30}$ and subcloned into pEGFP-N1 with NheI/PstI restriction enzymes.

medium. Six hours after the cell transfection, the medium was replaced with the pre-warmed complete medium.

To produce lipofectamine-plasmid DNA complex, $150 \mu \mathrm{l}$ of serum-free medium was mixed with $10 \mu \mathrm{l}$ of lipofectamine and incubated for 5 minutes at room temperature. Then, $150 \mu \mathrm{l}$ incomplete DMEM was mixed with $4 \mu \mathrm{g}$ of plasmids, added to lipofectamine solution, mixed gently, and incubated for 30 minutes at room temperature to form the DNA-lipofectamine complexes. After that, transfection complexes were added to each well and the medium was replaced after 6 hours of incubation at $37^{\circ} \mathrm{C}$ with pre-warmed DMEM, 10\% FBS and 1/100 penicillin/streptomycin. After 48 hours, transfection efficiency using TurboFect as well as 


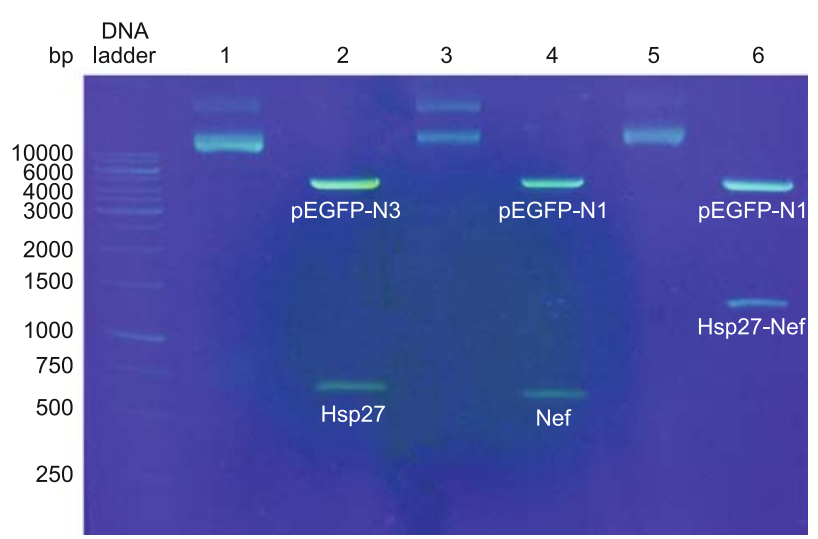

Fig. 2. Confirmation of the recombinant plasmids by double-digestion on gel electrophoresis: Lanes 1, 3, 5 represent the purified plasmids of pEGFP-Hsp27, pEGFP-Nef, pEGFP-Hsp-Nef on $1 \%$ agarose gel, respectively; Lanes 2, 4, 6 denote the double digested products using NheI/SalI for pEGFP-Hsp27 ( 720 bp), NheI/PstI for pEGFP-Nef ( $648 \mathrm{bp}$ ), and pEGFP-Hsp27-Nef ( $1368 \mathrm{bp}$ ), respectively.

lipofectamine was evaluated by fluorescent microscopy, flow cytometry, and western blotting.

\section{Transfection assay}

Fluorescent microscopy and Flow cytometry

Fluorescent microscopy and Flow cytometry were used to determine transfection efficiency. The levels of Nef-GFP, Hsp27GFP, Hsp27-Nef-GFP, and GFP protein expression was estimated after 48-h transfection using fluorescent microscopy (Envert Fluorescent Ceti, Korea) and quantified by a fluorescence-activated cell sorting (FACS) caliber flow cytometer (Partec, Germany). For flow cytometry analysis, the cells were harvested by trypsin and the cell
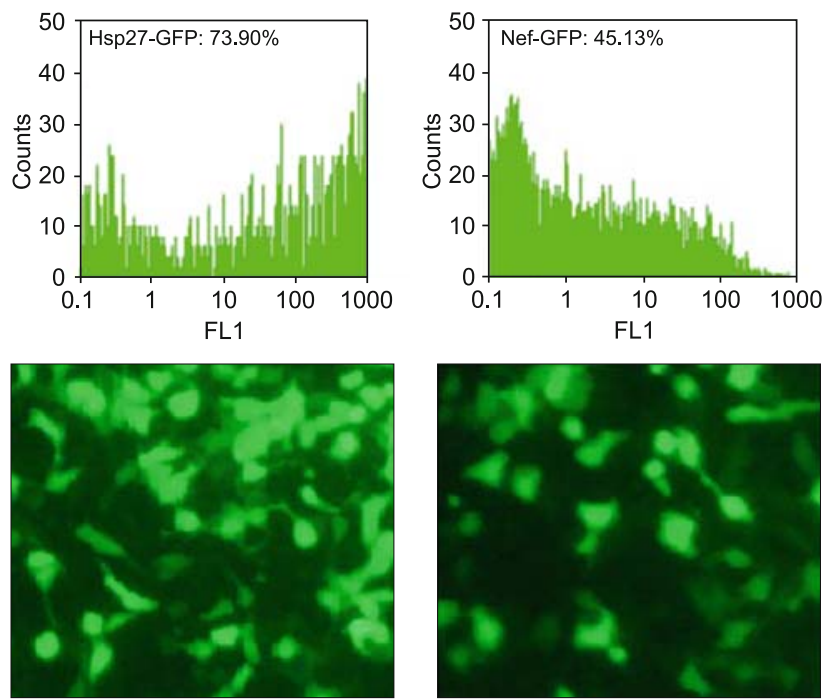

A

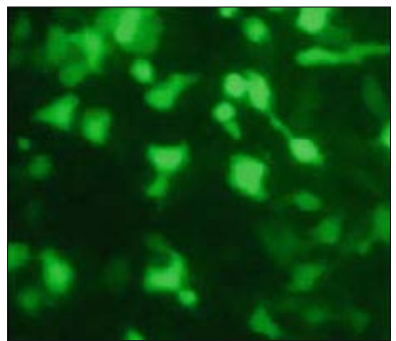

(B) pellets were resuspended in $1 \mathrm{ml} \mathrm{PBS}(\mathrm{pH}=7.4)$. The expression of fluorescent genes was measured in the FL1 channel using an excitation filter $(485 \mathrm{~nm})$ and emission filter $(535 \mathrm{~nm})$. The untransfected HEK-293T cells and transfected cells with pEGFP-N1 were used as negative and positive controls, respectively. 10,000 cells were counted in each analysis.

\section{Western blotting}

For western blotting, the cells were harvested by trypsin, and the cell pellets were resuspended in PBS. Total cellular proteins were solved in $6 \mathrm{X}$ sample buffer containing Tris- $\mathrm{HCl}(0.5 \mathrm{M})$, glycerol, SDS, and 2-Mercaptoethanol (2\%). The samples were separated on $12.5 \%$ acrylamide gel and transferred to nitrocellulose membrane. The membrane was incubated in blocking buffer (TBS 10X, $0.1 \%$ Tween20, BSA, Merck) and washed with TBS10X and $0.1 \%$ Tween 20 . Then, anti-GFP polyclonal antibody conjugated with horseradish peroxidase $(1: 10000 \mathrm{v} / \mathrm{v})$ was used to detect the proteins of interest in the presence of DAB substrate (Roche Diagnostics-Germany).

\section{Results}

\section{Confirmation of the DNA constructs}

The Hsp27 was correctly cloned in pEGFP-N3 as a $720 \mathrm{bp}$ fragment on $1 \%$ gel electrophoresis after digestion with NheI/SalI enzymes. Furthermore, the clear bands of $648 \mathrm{bp}$ for Nef gene and $1368 \mathrm{bp}$ for Hsp27-Nef fusion were appeared on gel electrophoresis after digestion of pEGFP-Nef and pEGFP-Hsp-Nef with NheI/ PstI enzymes as shown in Figures 1 and 2.

Transfection assay using fluorescent microscopy and flow cytometry

The DNA transfection efficiency of Turbofect and Lipofectamine was demonstrated by fluorescent microscopy and flow
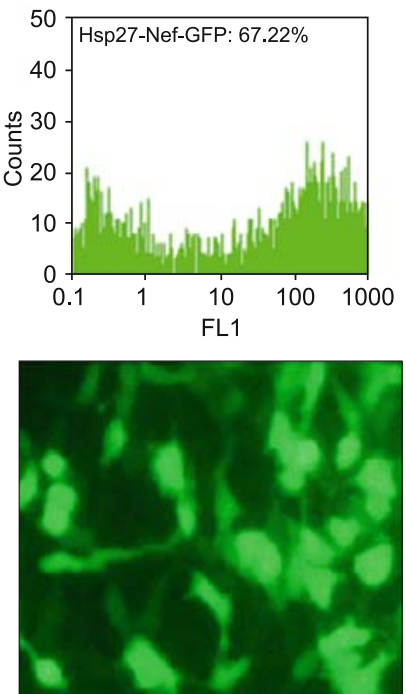

(C)
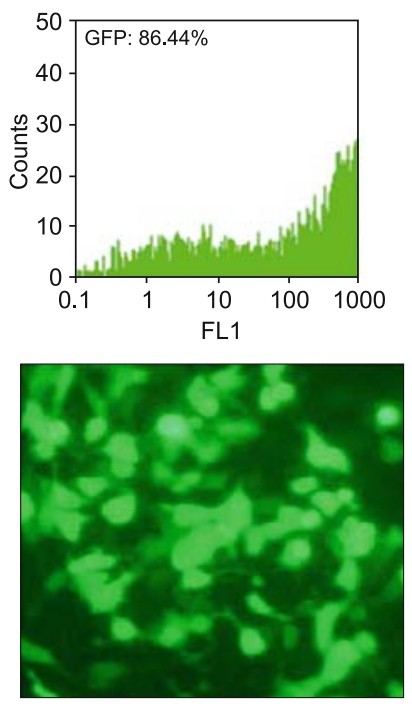

(D)

Fig. 3. Analysis of Hsp27-GFP (A), Nef-GFP (B), Hsp27-Nef-GFP (C), and GFP (D) expression in HEK-293T cells by Turbofect transfection reagent using Fluorescent microscopy and flow cytometry. The pEGFP-N1 (D) was used as a positive control. 


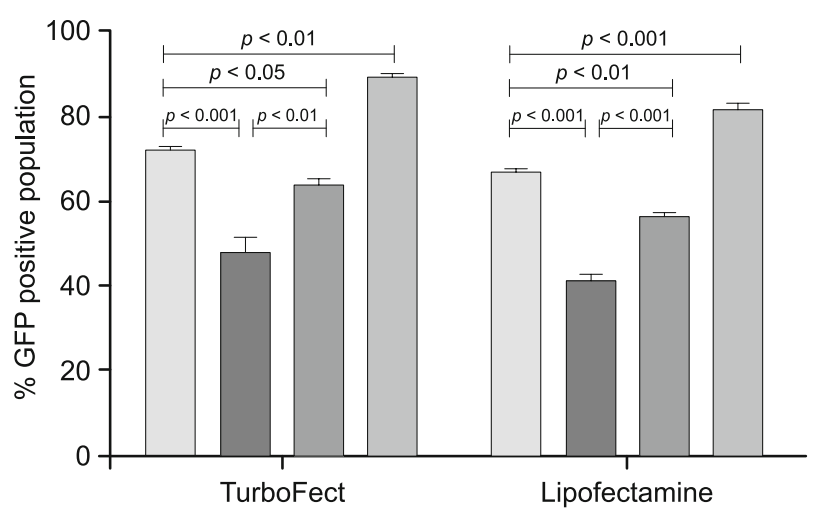

$\square$ Hsp27-GFP $\square$ Nef-GFP $\square$ Hsp27-Nef-GFP $\square$ GFP

Fig. 4. Comparison of TurboFect and Lipofectamine delivery: The transfection efficiency of TurboFect was significantly higher than that of Lipofectamine.

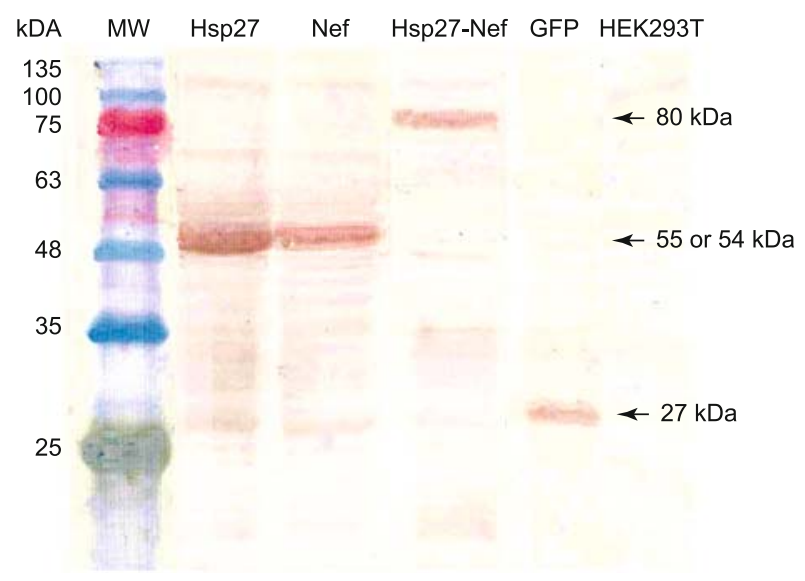

Fig. 5. Identification of protein expression in HEK-293T cells $48 \mathrm{~h}$ after transfection using western blot analysis. The expression of Hsp27GFP ( $54 \mathrm{kDa})$, Nef-GFP ( $53 \mathrm{kDa})$, and Hsp27-Nef-GFP ( $80 \mathrm{kDa})$ proteins was detected by an anti-GFP antibody as compared to the un-transfected cells. The GFP expression ( $27 \mathrm{kDa})$ was applied as a positive control. MW is the molecular weight marker.

cytometry as shown in Figure 3. Flow cytometry analysis showed that the permeability of pEGFP-Hsp27, pEGFP-Nef, and pEGFPHsp27-Nef using Turbofect transfection system was greater than that achieved with Lipofectamine $(p<0.05)$. These results were determined by the percentage of protein expression using GFP reporter marker. The transfection efficiency of Turbofect and Lipofectamine reagents was summarized in Figure 4. The percentage of Hsp27-GFP, Nef-GFP, Hsp27-Nef-GFP, GFP expression were $72.19 \pm 0.60,47.66 \pm 3.58,63.76 \pm 1.49,89.13 \pm 0.83$ for TurboFect system, and $66.72 \pm 0.80,41.36 \pm 1.38,56.20 \pm 0.65,81.39 \pm$ 1.45 for lipofectamine, respectively. Regarding these data, Hsp27 could increase Nef DNA delivery in the cells more significantly than Nef DNA alone using both TurboFect and lipofectamine transfection reagents $(\mathrm{p}<0.05)$.

\section{Western blotting}

Western blot analysis showed a successful expression of Hsp27-GFP, Nef-GFP, Hsp27-Nef-GFP, GFP proteins using antiGFP antibody (Fig. 5). In The data indicated clear bands of $\sim 54$ $\mathrm{kDa}, \sim 53 \mathrm{kDa}, \sim 80 \mathrm{kDa}$, and $\sim 27 \mathrm{kDa}$ for Hsp27-GFP, NefGFP, Hsp27-Nef-GFP, and GFP, respectively in western blotting.

\section{Discussion}

The main goal of the current study was to prepare the fusion of Hsp27 and Nef for the first time and evaluate its expression levels after transfection in mammalian cells using TurboFect and Lipofectamine delivery systems. In vitro transfection of different cell lines is an important challenge for evaluating the gene expression. To achieve the highest transfection efficiency, it is needed to assess various gene delivery systems. The ideal gene delivery systems should have high transfection efficiency and low toxicity in the cells (12). HIV-1 Nef is considered a key factor in HIV pathogenesis $(20,21)$. The studies showed that transfection of Nef gene in HEK- 293 was performed by a liposomal transfection agent named Effectene, exhibiting the ability of this system to transport Nef into the cell (22). Another study showed that HIV-1 Nef gene was transfected into human coronary arterial endothelial cells (HCAEC) and Jurkat cells with lipofectamine LTX reagent, and the transfection efficiency was $70 \%$ and $20 \%$, respectively (23). Among other lipid-based transfection reagents, flow cytometry analysis showed that GFP expression in different myeloma cell lines with Lipofectamine 2000 has higher efficiency as compared to LyoVec lipolex. Efficiency rates of pEGFP-N1 transfection in HEK293-FT, NS0 and Ag8 were efficiently higher than those of SP2/0, NS1 and P3U1, which were hardly transfected by these reagents (24). Our results demonstrated that cationic TurboFect polymer was more effective than lipofectamine, a cationic lipid, for transfection and expression of Hsp27, Nef, and Hsp27-Nef genes into HEK-293T cells. Furthermore, the analysis of HIV-1 Nef and Hsp27-Nef delivery using TurboFect and lipofectamine showed that Hsp27 could increase Nef expression in HEK-293T cells. Indeed, transfection of Hsp27 gene was also higher than that of Nef gene using both delivery systems. A study indicated that the efficiency of HIV-1 MPER-V3 gene expression in HEK293 T using Lipofectamine and Turbofect was $55 \%$ and $65 \%$, respectively, indicating higher efficiency of TurboFect reagent (25). Physical methods such as electroporation were also compared with chemical methods of DNA transfer. For example, lipofectamine showed greater efficiency in transfection $(\sim 42.4 \%)$ than in electroporation $(\sim 37.3 \%)$ in an adherent human lung cancer cell line (Mehr-80 cells). In addition, both lipofectamine and electroporation reagents indicated higher transfection efficiency than other techniques such as SuperFect and DEAE-dextran reagents (26). Similar to our study, Hauser and colleagues demonstrated that the transfection of Hsp70-HPVE7 fusion into HEK-293A cells by Lipofectamine Plus was successful in comparison with each gene, alone suggesting the use of Hsps for increasing the potency of DNA vaccine in vivo (27). Another study also represented that transfection of Hsp27 with lipofectamine2000 in RGC-5 cells 
was significantly elevated ( $\sim 25$-fold) under stress conditions as compared to untransfected cells (28).

\section{Conclusion}

Although different systems were used to transfer genes with various efficiency, in this study we showed that the efficiency of TurboFect transfection reagent of genes is higher than that of lipofectamine, which makes the former a more suitable tool for in vitro delivery in the future. Our observation showed the efficiency of Hsp27 to increase Nef delivery in DNA vaccines against HIV1 infections. However, further study is required to investigate the Hsp27 mechanisms for enhancing gene delivery.

\section{References}

1. Clapham PR, McKnight A. HIV-1 receptors and cell tropism. Bri Med Bull 2001; 58: 43-59.

2. Geijtenbeek TB, Kwon DS, Torensma R et al. DC-SIGN, a dendritic cell-specific HIV-1-binding protein that enhances trans-infection of T cells. Cell 2000; 100 (5): 587-597.

3. Li G, Piampongsant S, Faria NR et al. An integrated map of HIV genome-wide variation from a population perspective. Retrovirology 2015; 12 (1): 1 .

4. Mwimanzi P, Markle TJ, Ogata Y et al. Dynamic range of Nef functions in chronic HIV-1 infection. Virology 2013; 439 (2): 74-80.

5. Stumptner-Cuvelette P, Morchoisne S, Dugast M et al. HIV-1 Nef impairs MHC class II antigen presentation and surface expression. P Natl Acad Sci USA 2001; 98 (21): 12144-12149.

6. Dikeakos JD, Atkins KM, Thomas L et al. Small molecule inhibition of HIV-1-induced MHC-I down-regulation identifies a temporally regulated switch in Nef action. Mol Biol Cell 2010; 21 (19): 3279-3292.

7. Peterlin BM, Trono D. Hide, shield and strike back: how HIV-infected cells avoid immune eradication. Nat Re. Immunol 2003; 3 (2): 97-107.

8. Rosa A, Chande A, Ziglio S et al. HIV-1 Nef promotes infection by excluding SERINC5 from virion incorporation. Nature 2015; 526 (7572): 212-217.

9. Watkins RL, Foster JL, Garcia JV. In vivo analysis of Nef's role in HIV-1 replication, systemic T cell activation and CD4+ T cell loss. Retrovirology 2015; 12 (1): 61.

10. Gurunathan S, Klinman DM, Seder RA. DNA vaccines: immunology, application, and optimization*. Annu Rev Immunol 2000; 18: 927-974.

11. Khosravi-Darani K, Mozafari MR, Rashidi L, Mohammadi M. Calcium based non-viral gene delivery: an overview of methodology and applications. Acta Med Iran 2010; 48 (3): 133-141.

12. Jin L, Zeng X, Liu M, Deng Y, He N. Current progress in gene delivery technology based on chemical methods and nano-carriers. Theranostics 2014; 4 (3): 240-255.

13. Segal BH, Wang XY, Dennis CG et al. Heat shock proteins as vaccine adjuvants in infections and cancer. Drug Discov Today 2006; 11 (11-12): 534-540.
14. Garrido C, Gurbuxani S, Ravagnan L, Kroemer G. Heat shock proteins: endogenous modulators of apoptotic cell death. Biochem Biophys Res Commun 2001; 286 (3): 433-442.

15. Calderwood SK, Gong J, Murshid A. Extracellular HSPs: The Complicated Roles of Extracellular HSPs in Immunity. Front Immunol 2016; 7: 159 .

16. Gusev NB, Bukach OV, Marston SB. Structure, properties, and probable physiological role of small heat shock protein with molecular mass 20 kD (Hsp20, HspB6). Biochemistry Mosc 2005; 70 (6): 629-637.

17. Alford KA, Glennie S, Turrell BR, Rawlinson L, Saklatvala J, Dean JL. Heat shock protein 27 functions in inflammatory gene expression and transforming growth factor-beta-activated kinase-1 (TAK1)-mediated signaling. J. Biol. Chem 2007; 282 (9): 6232-6241.

18. Salari S, Seibert T, Chen YX et al. Extracellular HSP27 acts as a signaling molecule to activate NF-kappaB in macrophages. Cell Stress Chaperones 2013; 18 (1): 53-63.

19. Jin C, Cleveland JC, Ao L et al. Human myocardium releases heat shock protein 27 (HSP27) after global ischemia: the proinflammatory effect of extracellular HSP27 through toll-like receptor (TLR)-2 and TLR4. Mol Med 2014; 20: 280-289.

20. Lundquist CA, Tobiume M, Zhou J, Unutmaz D, Aiken C. Nefmediated downregulation of CD4 enhances human immunodeficiency virus type 1 replication in primary T lymphocytes. J Virol 2002; 76 (9): 4625-4633.

21. Abraham L, Fackler OT. HIV-1 Nef: a multifaceted modulator of T cell receptor signaling. Cell Commun Signal 2012; 10 (1): 39.

22. Raymond AD, Campbell-Sims TC, Khan M et al. HIV Type $1 \mathrm{Nef}$ is released from infected cells in CD45 (+) microvesicles and is present in the plasma of HIV-infected individuals. AIDS Res Hum Retroviruses 2011; 27 (2): 167-178.

23. Wang T, Green LA, Gupta SK et al. Transfer of intracellular HIV Nef to endothelium causes endothelial dysfunction. PLoS One 2014; 9 (3): e91063.

24. Shabani M, Hemmati S, Hadavi R et al. Optimization of Gene Transfection in Murine Myeloma Cell Lines using Different Transfection Reagents. Avicenna J Med Biotechnol 2010; 2 (3): 123-130.

25. Bolhassani A, Kardani K, Vahabpour R et al. Prime/boost immunization with HIV-1 MPER-V3 fusion construct enhances humoral and cellular immune responses. Immunol Lett 2015; 168 (2): 366-373.

26. Salimzadeh L, Jaberipour M, Hosseini A, Ghaderi A. Non-viral transfection methods optimized for gene delivery to a lung cancer cell line. Avicenna J Med Biotechnol 2013; 5 (2): 68-77.

27. Hauser H, Shen L, Gu QL, Krueger S, Chen SY. Secretory heatshock protein as a dendritic cell-targeting molecule: a new strategy to enhance the potency of genetic vaccines. Gene Ther 2004; 11 (11): 924-932.

28. Whitlock NA, Lindsey K, Agarwal N, Crosson CE, Ma J-X. Heat Shock Protein 27 Delays Ca2+-Induced Cell Death in a Caspase-dependent and -independent Manner in Rat Retinal Ganglion Cells. Invest Ophthalmol Vis Sci 2005; 46 (3): 1085-1091.

Received February 7, 2017. Accepted February 25, 2017. 